EPJ Web of Conferences 52, 07005 (2013)

DOI: $10.1051 /$ epjconf/20135207005

(C) Owned by the authors, published by EDP Sciences, 2013

\title{
Evidence for some new physical process in ultrahigh-energy collisions
}

\author{
Glennys R. Farrar ${ }^{1, a}$, Jeff Allen ${ }^{1}$ \\ ${ }^{1}$ New York University, USA
}

\begin{abstract}
Combining the published Pierre Auger Observatory measurements of the longitudinal and lateral properties of UHE atmospheric showers is shown to point to an unforeseen change in the nature of particle interactions at ultrahigh energy. A "toy model" of UHE proton-Air interactions is presented which provides the first fully consistent description of air shower observations. While fundamentally phenomenological, the model is based on considerations of how the normal vacuum of QCD might be vaporized and chiral symmetry restored by the extreme energy densities produced in UHE collisions.
\end{abstract}

Slides

The slides of the talk can be found on the website of the symposium ISVHECRI 2012:

https://indico.desy.de/conferenceOtherViews.py?view=standard\&confId $=4594$

${ }^{a}$ gf $25 @$ nyu.edu 